\title{
Treatment Patterns of Lipid-Lowering Therapy in Patients with Coronary Artery Disease Aged Above and Below 75 Years: A Retrospective Cross-Sectional Study of 1500 Patients in a Tertiary Care Referral Center in Germany
}

\author{
Iryna Dykun ${ }^{1}$ (D) Daniela Wiefhoff ${ }^{1} \cdot$ Matthias Totzeck $^{1} \cdot$ Fadi Al-Rashid $^{1} \cdot$ R. Alexander Jánosi ${ }^{1} \cdot$ Stefanie Hendricks $^{1}$. \\ Tienush Rassaf ${ }^{1} \cdot$ Amir A. Mahabadi $^{1}$
}

Published online: 28 May 2020

(c) The Author(s) 2020

\begin{abstract}
Introduction Lipid-lowering therapy of elderly patients with coronary artery disease (CAD) inherits a medical challenge, as these patients experience a higher absolute risk reduction but may be more prone to side effects. We aimed to evaluate the treatment patterns in lipid-lowering therapy, comparing CAD patients above versus below 75 years of age.

Methods We retrospectively included patients with known CAD admitted to the West German Heart and Vascular Center. Low-density lipoprotein cholesterol (LDL-C) levels and intensity of statin therapy (based on dosage and type of statin) were assessed from all available hospital records.

Results We included 1500 patients (mean age $68.4 \pm 11.2$ years, $75.7 \%$ male) from 813 referring treating primary care physicians in 98 cities of Germany in our analysis. A total of 982 patients were $<75$ years of age, whereas 518 were $\geq 75$ years of age. LDL-C levels did not differ between age groups ( $\geq 75: 96.0 \pm 35.1 \mathrm{mg} / \mathrm{dl} ;<75: 98.9 \pm 35.8 \mathrm{mg} / \mathrm{dl}, p=0.13)$. Simvastatin was most frequently prescribed in both age groups ( $54.9 \%$ vs. $50.7 \%$ for age $\geq 75$ vs. $<75$ years, $p=0.16$ ), followed by atorvastatin $(31.6 \%$ vs. $33.3 \%, p=0.53)$. Elderly patients received slightly lower statin doses as compared to patients $<75$ years of age $(28.8 \pm 12.8 \mathrm{mg}$ vs. $31.4 \pm 13.7 \mathrm{mg}, p=0.0007)$. Interestingly, patients $\geq 75$ years of age achieved LDL-C $<70 \mathrm{mg} /$ dl slightly more frequently than younger patients $(24.0 \%$ vs. $20.1 \%, p=0.09)$, while only a minority had LDL-C $<55 \mathrm{mg} / \mathrm{dl}$ in both age groups. Excluding patients with myocardial infarction at presentation, creatine kinase levels were not relevantly different between age groups $(131.9 \pm 450.0 \mathrm{U} / \mathrm{l}$ vs. $127.5 \pm 111.4 \mathrm{U} / 1, p=0.78)$.

Conclusion Patients $\geq 75$ years of age receive lower doses of statin therapy and reach slightly lower LDL-C levels. However, the majority of elderly patients miss current recommendations regarding LDL-C thresholds.
\end{abstract}

\section{Introduction}

With increasing survival, the proportion of elderly people in the general population continues to grow. However, the elderly carry a disproportionate burden of atherosclerotic cardiovascular disease $[1,2]$. Statin therapy for secondary prevention is established for risk reduction in patients with coronary artery disease (CAD) and has proven its benefit also in elderly cohorts [3, 4]. In addition to statin therapy,

Iryna Dykun

Iryna.dykun@uk-essen.de

1 Department of Cardiology and Vascular Medicine, West German Heart and Vascular Center, University of DuisburgEssen, Hufelandstr. 55, 45147 Essen, Germany ezetimibe and proprotein convertase subtilisin/kexin type 9 (PCSK-9) inhibitors have been introduced within the last decade, lowering both low-density lipoprotein cholesterol (LDL-C) and the incidence of major cardiovascular events in secondary prevention [5, 6]. Available data suggest that given the overall higher absolute event rate in elderly cohorts, these patients show a higher risk reduction and lower number needed to treat [7].

Despite the evidence supporting its clinical benefits, statin treatment is often discontinued for various reasons, such as concomitant disease and perceptions of risk versus benefit $[4,8-10]$. However, data on treatment patterns and cholesterol profiles in patients above 75 years of age are lacking. To provide information regarding the effect of aging on selection, dosing, and effects of lipid-lowering drugs, 


\section{Key Points}

Lipid-lowering therapy of elderly patients with coronary artery disease (CAD) inherits a medical challenge, as these patients experience higher absolute risk reduction but may be more prone to side effects.

In the present study, we evaluated the treatment patterns in lipid-lowering therapy, comparing CAD patients above and below 75 years of age.

While elderly patients receive lower doses of statin therapy and reach slightly lower low-density lipoprotein cholesterol (LDL-C) levels, the majority of elderly patients miss current recommendations regarding LDL-C thresholds.

we set out to evaluate treatment patterns of lipid-lowering therapy and its success in achieving LDL-C targets in a realworld, cross-sectional cohort of patients with manifest CAD, comparing those above and below 75 years of age.

\section{Methods}

\subsection{Study Cohort}

We retrospectively enrolled patients $\geq 18$ years old with known CAD (diagnosis at least 30 days prior to presentation) who received assessment of cholesterol levels and medication for clinical indications in the years between 2009 and 2016. Patients had to be on stable medical therapy for at least 30 days, including stable lipid-lowering therapy. Patients were randomly selected from hospital admissions and included both elective and emergency admissions at the West German Heart and Vascular Center, Essen. Of these patients, $24.1 \%$ were hospitalized due to an acute coronary syndrome (ACS), 37.4\% due to stable CAD, and $38.5 \%$ due to a non-coronary reason. Patients at each time point were not identical. Patients with low-density lipoprotein (LDL) apheresis, end-stage renal disease, familial hypercholesterolemia, and prior medical documentation of statin intolerance were excluded from the analysis. The study protocol conforms to the ethical guidelines of the 1975 Declaration of Helsinki, as reflected in a priori approval by the institution's human research committee (17-7458-BO).

\subsection{Risk Factors and Clinical Diagnosis}

Presence of known CAD manifestation was assessed from all available hospital records and defined as previous revascularization therapy, at least 30 days prior to the present admission. Cholesterol levels, demographic characteristics, cardiovascular risk factors, and medical therapy were assessed from available patient records. Statin therapy was categorized as low, moderate, and high intensity according to the 2013 American College of Cardiology/American Heart Association (ACC/AHA) definitions [3]. Serum LDL-C levels were categorized as meeting or missing European Society of Cardiology (ESC) guidelines according to recommendations at different time points $(<100 \mathrm{mg} / \mathrm{dl}$ for $2008,<70 \mathrm{mg} / \mathrm{dl}$ for 2012 , and $<55 \mathrm{mg} / \mathrm{dl}$ for 2019 [11-13].

\subsection{Statistical Analysis}

The baseline characteristics are presented as mean \pm standard deviation for continuous variables and as frequencies and percentages for categorical variables and are stratified by age group. A two-sided $t$ test was used for normally distributed continuous variables, Wilcoxon rank-sum tests were used for non-normally distributed continuous variables, and Fisher's exact test or Chi-squared test were used for categorical variables for comparisons of baseline characteristics at first versus last time points. The frequencies of patients according to LDL-C groups and statin intensity are stratified by age group. Difference in frequency of accordance to ESC recommendations and time points were compared using Fisher's exact test, comparing the first to the last period. All analyses were performed using SAS software (version 9.2, SAS Institute Inc.). A $p$ value of $<0.05$ indicated statistical significance.

\section{Results}

\subsection{Baseline Characteristics}

A total of 1500 patients (mean age $68.4 \pm 11.2$ years, $75.7 \%$ male) from 813 referring primary care physicians in 98 cities of Germany were included in our analysis. Overall, 982 patients were $<75$ years of age, whereas 518 were $\geq 75$ years of age. Patient characteristics, stratified by age group, are shown in Table 1. Elderly patients were more likely to be hospitalized due to a non-coronary reason $(47.5 \%$ vs. $33.8 \%$, for patients $\geq 75$ vs. $<75$ years of age, respectively, $p<0.001)$, followed by stable CAD (32.6\% vs. $39.9 \%$, $p<0.001)$. Older patients were less likely male $(68.0 \%$ vs. $79.8 \%, p<0.0001)$ and had a lower body mass index (BMI) $\left(26.8 \mathrm{~kg} / \mathrm{m}^{2}\right.$ vs. $\left.28.4 \mathrm{~kg} / \mathrm{m}^{2}, p<0.0001\right)$, and were less likely to be current smokers $(7.3 \%$ vs. $18.8 \%, p<0.0001$, for patients $\geq 75$ vs. $<75$ years of age, respectively). The rate of hypertension (95.6\% vs. $95.2 \%, p=0.03)$ and the rate of diabetes $(38.2 \%$ vs. $32.5 \%, p=0.02)$ were high in both age groups, but slightly higher in elderly patients. 


\subsection{Lipid Profile in Patients $\geq 75$ Versus $<75$ Years of Age}

LDL-C levels were not significantly different between age groups ( $\geq 75$ years: $96.0 \pm 35.1 \mathrm{mg} / \mathrm{dl} ;<75$ years: $98.9 \pm 35.8 \mathrm{mg} / \mathrm{dl}, p=0.13)$. In contrast, elderly patients had higher HDL-cholesterol levels $(49.9 \pm 15.0 \mathrm{mg} / \mathrm{dl}$ vs. $46.8 \pm 15.2, p=0.0002)$ and markedly lower triglycerides $(135.6 \pm 89.9 \mathrm{mg} / \mathrm{dl}$ vs. $171.4 \pm 124.6 \mathrm{mg} / \mathrm{dl}, p<0.0001)$.

When evaluating achievement rates of LDL-C targets as set by ESC guidelines, we observed that $7.6 \%$ of the cohort had LDL-C $<55 \mathrm{mg} / \mathrm{dl}$ (2019 guidelines), $21.5 \%$ had LDLcholesterol $<70 \mathrm{mg} / \mathrm{dl}$ (2012 guidelines), and $57.1 \%$ had LDL-C $<100 \mathrm{mg} / \mathrm{dl}$ (2008 guidelines). Interestingly, elderly patients more frequently reached the LDL-C threshold of $<55 \mathrm{mg} / \mathrm{dl}$ as recommended by current ESC guidelines (9.7\% vs. $7.0 \%$ for $\geq 75$ vs. $<75$ years of age, $p=0.03$ ). In contrast, there was a non-significant difference in achieving LDL-C goals as defined by 2012 ESC guidelines when comparing both age groups, and no relevant difference with regard to LDL-C $<100$ as the threshold (Fig. 1). Patients hospitalized due to an ACS had significantly higher LDL-C levels as compared to patients without ACS at presentation (ACS: $103.5 \pm 38.7 \mathrm{mg} / \mathrm{dl}$, non-ACS $96.1 \pm 34.3 \mathrm{mg} / \mathrm{dl}$, $p=0.001)$. This effect was present for patients $<75$ years of age (ACS: $106.9 \pm 38.1 \mathrm{mg} / \mathrm{dl}$, non-ACS $96.0 \pm 34.5 \mathrm{mg} /$ $\mathrm{dl}, p<0.0001$ ), but not for elderly patients (ACS: $95.2 \pm 39.2 \mathrm{mg} / \mathrm{dl}$, non-ACS $96.2 \pm 34.1 \mathrm{mg} / \mathrm{dl}, p=0.8$ ).

\subsection{Trend in Statin Use and Dose in Elderly}

The most common statin used was simvastatin in both age groups $(50.7 \%$ vs. $54.9 \%$ for age $\geq 75$ vs. $<75$ years, $p=0.16)$, followed by atorvastatin $(33.3 \%$ vs. $31.6 \%$,

Table 1 Study sample characteristics

\begin{tabular}{|c|c|c|c|c|}
\hline & All patients $(n=1500)$ & $\geq 75(n=518)$ & $<75(n=982)$ & $p$ value \\
\hline Age, years & $68.4 \pm 11.2$ & $79.8 \pm 3.9$ & $62.4 \pm 8.8$ & $<0.0001$ \\
\hline $\mathrm{BMI}\left(\mathrm{kg} / \mathrm{m}^{2}\right)$ & $27.9 \pm 4.9$ & $26.8 \pm 4.3$ & $28.4 \pm 5.0$ & $<0.0001$ \\
\hline Sex, $n$ (\% male $)$ & $1136(75.7)$ & $352(68.0)$ & $784(79.8)$ & $<0.0001$ \\
\hline Total cholesterol (mg/dl) & $170.0 \pm 45.3$ & $167.1 \pm 41.6$ & $171.5 \pm 47.1$ & 0.06 \\
\hline HDL-cholesterol (mg/dl) & $47.8 \pm 15.2$ & $49.9 \pm 15.0$ & $46.8 \pm 15.2$ & 0.0002 \\
\hline LDL-cholesterol (mg/dl) & $97.9 \pm 35.6$ & $96.0 \pm 35.1$ & $98.9 \pm 35.8$ & 0.13 \\
\hline Triglyceride (mg/dl) & $159.0 \pm 115.1$ & $135.6 \pm 89.9$ & $171.4 \pm 124.6$ & $<0.0001$ \\
\hline hs-CRP & $0.36 \pm 1.0$ & $0.45 \pm 1.2$ & $0.32 \pm 0.86$ & 0.1 \\
\hline $\mathrm{BNP}$ & $224.3 \pm 427.8$ & $295.3 \pm 527.4$ & $186.7 \pm 359.0$ & $<0.0001$ \\
\hline GFR & $58.4 \pm 17.8$ & $53.4 \pm 17.0$ & $61.0 \pm 17.7$ & $<0.0001$ \\
\hline Statins, $n(\%)$ & $1332(88.8)$ & $438(84.6)$ & $894(91.0)$ & 0.0002 \\
\hline Non-statin, $n(\%)$ & $60(4.0)$ & $44(4.5)$ & $16(1.6)$ & 0.34 \\
\hline Hypertension, $n(\%)$ & $1430(95.3)$ & $495(95.6)$ & $935(9)$ & 0.03 \\
\hline Diabetes, $n(\%)$ & $517(34.5)$ & $198(38.2)$ & $319(32.5)$ & 0.02 \\
\hline Family history of premature CHD, $n(\%)$ & $461(34.5)$ & $114(27.8)$ & $347(35.3)$ & $<0.0001$ \\
\hline Smoking, $n(\%)$ & & & & $<0.0001$ \\
\hline Current & $223(14.9)$ & $38(7.3)$ & $185(18.8)$ & \\
\hline Former & $441(29.4)$ & $126(24.3)$ & $315(32.1)$ & \\
\hline Hospitalization reason, $n(\%)$ & & & & $<0.0001$ \\
\hline Non-coronary & $578(38.5)$ & $246(47.5)$ & $332(33.8)$ & \\
\hline Stable CAD & $561(37.4)$ & $169(32.6)$ & $392(39.9)$ & \\
\hline Unstable CAD & $268(17.9)$ & $74(14.3)$ & $194(19.8)$ & \\
\hline NSTEMI & $78(5.2)$ & $23(4.4)$ & $55(5.6)$ & \\
\hline STEMI & $15(1.0)$ & $6(1.2)$ & $9(0.9)$ & \\
\hline Previous CABG, $n(\%)$ & $522(34.8)$ & $210(40.5)$ & $312(31.8)$ & 0.0005 \\
\hline Previous STEMI, $n(\%)$ & $399(26.6)$ & $113(21.8)$ & $286(29.1)$ & 0.003 \\
\hline
\end{tabular}

Data are presented as mean and standard deviation for continuous variables and as frequency and percentages for categorical variables $B M I$ body mass index, $B N P$ brain natriuretic peptide test, $C A B G$ coronary artery bypass graft, $C A D$ coronary artery disease, $C H D$ coronary heart disease, $G F R$ glomerular filtration rate, $H D L$ high-density lipoprotein, $h s$ - $C R P$ high-sensitivity C-reactive protein, $L D L$ low-density lipoprotein, NSTEMI non-ST-elevation myocardial infarction, STEMI ST-elevation myocardial infarction 
$p=0.53)$ and rosuvastatin $(1.7 \%$ vs. $6.1 \%, p=0.0002$, Fig. 2a). In contrast, non-statin lipid-lowering therapy was infrequently used for both age groups $(4.5 \%$ vs. $1.6 \%$, $p=0.21$ ). Elderly patients received significantly lower doses of statins $(28.8 \pm 12.8 \mathrm{mg}$ vs. $31.4 \pm 13.7 \mathrm{mg}, p=0.0007$, Fig. 2b). Figure 3 depicts the frequency of no statin and low-, medium-, and high-intensity statin therapy in 2009-2010, 2012-2013, and 2015-2016, stratified by age group. While in 2009-2010, treatment regimens between patients $<75$ and $\geq 75$ years of age did not significantly differ, a progressive trend towards less-intensive treatment protocols in the elderly was observed at later time points. This was especially prominent in the group of coronary heart disease patients receiving no statin therapy, which reached one out of five patients $\geq 75$ years of age in 2015-2016.

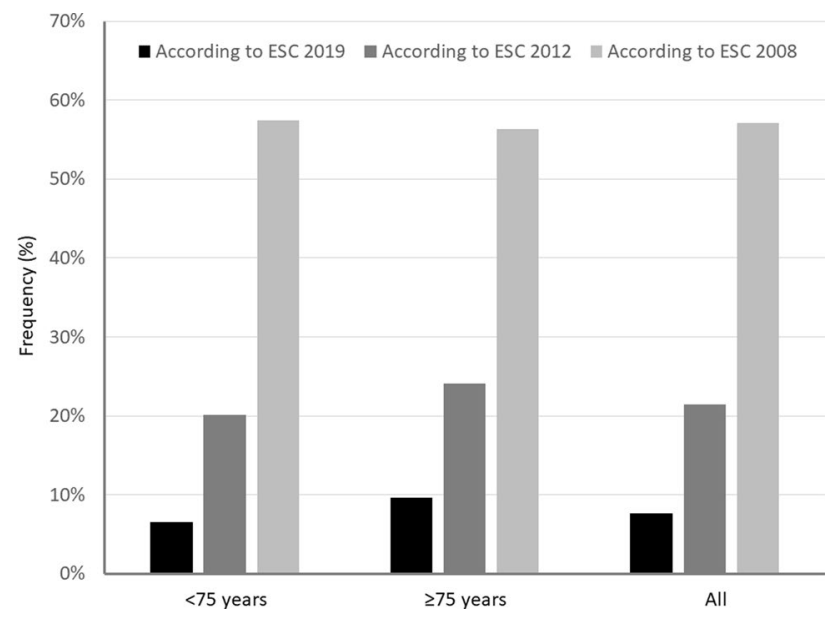

Fig. 1 Achievement of LDL-C targets according to 2019 (LDL-C $<55 \mathrm{mg} / \mathrm{dl}$ ), 2012 (LDL-C $<70 \mathrm{mg} / \mathrm{dl}$ ), and 2008 (LDL $<100 \mathrm{mg} / \mathrm{dl}$ ) ESC guidelines, stratified by age $\geq 75$ vs. $<75$ years. ESC European Society of Cardiology, $L D L-C$ low-density lipoprotein cholesterol

\subsection{Creatine Kinase and High-Sensitivity C-Reactive Protein Levels Under Statin Therapy}

Excluding patients with myocardial infarction at presentation, creatine kinase levels were not relevantly different between age groups $(131.9 \pm 450.0 \mathrm{U} / 1$ vs. $127.5 \pm 111.4 \mathrm{U} / \mathrm{l}$, $p=0.78$ ). Likewise, excluding patients with signs of systemic inflammation, high-sensitivity C-reactive protein levels did not differ when comparing patients $\geq 75$ and $<75$ years of age $(0.15 \pm 0.12 \mathrm{mg} / \mathrm{dl}$ vs. $0.14 \pm 0.12 \mathrm{mg} / \mathrm{dl}$, $p=0.33$ ).

\section{Discussion}

Evaluating lipid-lowering treatment in a large real-world registry with 1500 patients from 813 primary care physicians in 98 German cities, we observed that patients $\geq 75$ years of age receive lower doses of statin therapy and reached slightly lower LDL-C levels. The majority of patients with known CAD miss current recommendations regarding LDL-C thresholds. But despite lower treatment intensity, elderly patients more frequently reached the strict goals regarding LDL-C as defined by current ESC guidelines. However, as a trend over time, we observed an increase in high-intensity statin therapy also in the elderly group. Interestingly, in this observational cross-sectional analysis, we observed no signs of a higher frequency of statin-induced myopathy in the elderly. While the frequency of patients without statin therapy was higher in the elderly group, we cannot rule out that therapy was discontinued due to potential statin-induced adverse events.

In 2011, the ESC guidelines changed the treatment goals for LDL-C for patients with CAD manifestation. In the present analysis, we investigated how this recommendation impacted LDL-C levels 1-2 years (2012-2013)
A

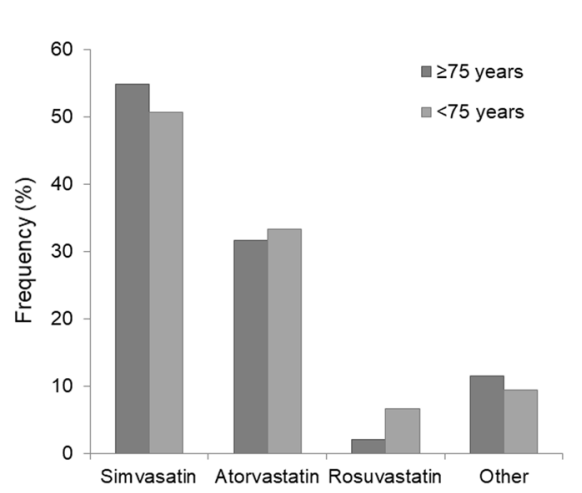

B

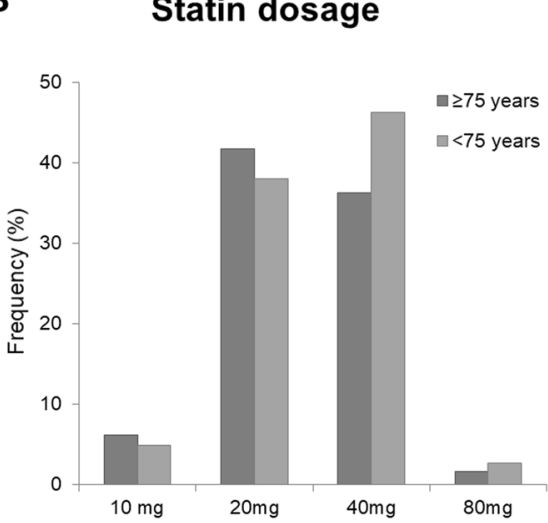

C

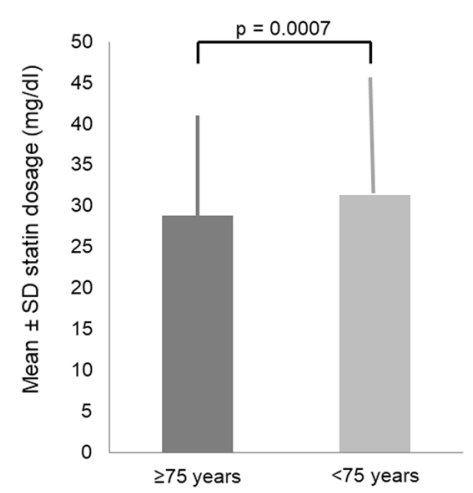

Fig. 2 Frequency of type of statin (a) and statin dosage (b), as well as mean statin dosage $( \pm \mathrm{SD})$ (c) in patients with known CAD, stratified by age $\geq 75$ vs. $<75$ years. $C A D$ coronary artery disease, $S D$ standard deviation 

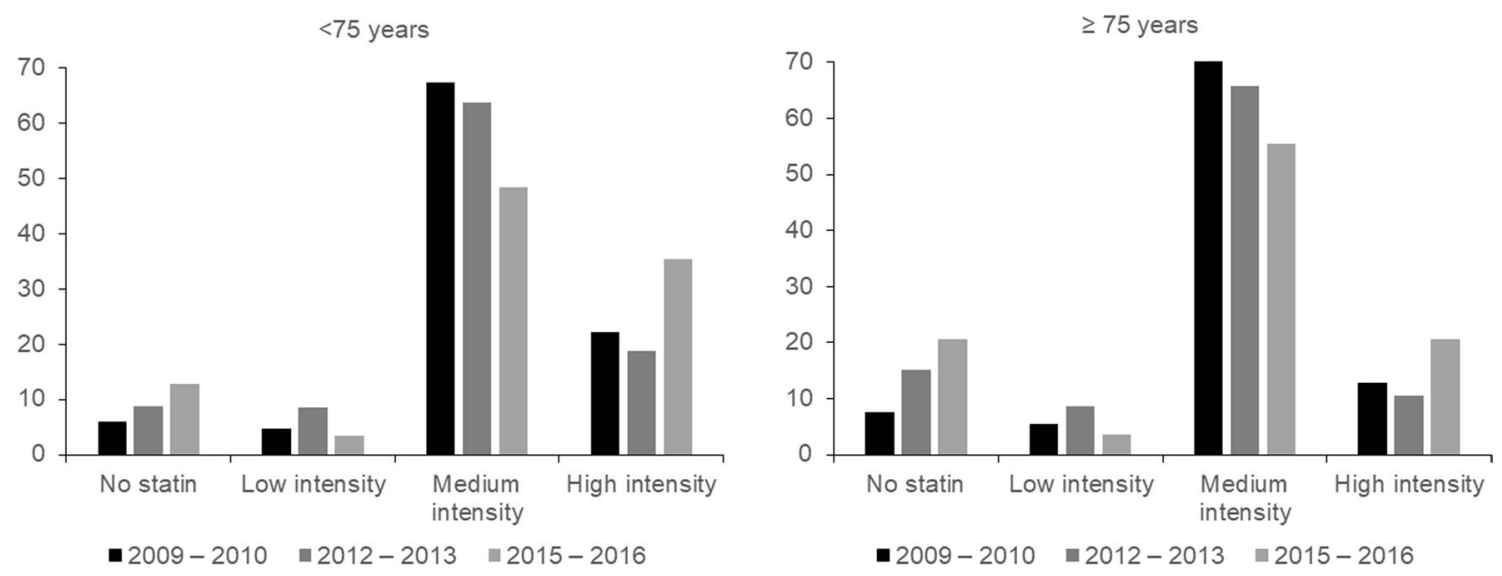

Fig. 3 Frequency (\%) of low-, medium-, and high-intensity statin therapy in patients $<75$ vs. $\geq 75$ years of age, stratified by time point of assessment according to the 2013 ACC/AHA definition [3]. ACC/AHA American College of Cardiology/American Heart Association

as well as 4-5 years (2015-2016) later, as compared to LDL-C levels before the change in LDL-C goals (2009-2010). Our finding of a poor achievement in current treatment goals is in accordance with existing literature for other European cohorts, demonstrating that also in very high-risk cohorts, median LDL-C levels of $>70 \mathrm{mg} / \mathrm{dl}$ or even $>100 \mathrm{mg} / \mathrm{dl}$ frequently occur [14-16]. This finding was also reflected in the USA, where a trend analysis in the use of statins for secondary prevention showed nearly flat trends among the patients with a history of atherosclerotic cardiovascular disease during the 3 years that followed the publication of the 2013 ACC/AHA guideline. However, in this analysis, the age groups with the highest odds of statin use were 76- to 79-year-olds and 65- to 69-year-olds [17]. Also, in a Chinese population-based community analysis, the SHECHS study showed poor control of high cholesterol levels in older individuals with established cardiovascular disease using mostly low doses of statins, and a high proportion of these subjects did not achieve the LDL-C target level [18]. All these studies underline the unmet need of successful lipid control in this high-risk population.

Overall, there has been intensified debate on the use of statins in the older population, related to concerns about adding another medication, consideration of nonadherence, and discussion of metabolic (diabetes mellitus) and musculoskeletal (myalgia, myositis, and the very rare rhabdomyolysis) issues, medication interactions, major organ effects (liver and kidney), and memory concerns [19]. In contrast, due to the higher absolute risk in elderly cohorts, the absolute risk reduction of preventive strategies is higher in this age group [6, 7, 20, 21]. Likewise, individual randomized trials and meta-analysis of statin therapy have reported significant cardiovascular risk reductions among elderly participants using statins as well as non-statin lipid-lowering therapy $[10,11,22-26]$.
A recently published meta-analysis of 28 randomized controlled trials by the Cholesterol Treatment Trialists' Collaboration focused on age-dependent effects of statin therapy and included 14,483 (8\%) of 186,854 participants older than 75 years at randomization, with a median followup duration of 4.9 years. The authors observed a significant reduction in major vascular events in all age groups; although proportional reductions in major vascular events diminished slightly with age, this trend was not statistically significant. There is less definitive direct evidence of benefit in the primary prevention setting among patients older than 75 years, but evidence supports the use of statin therapy in older people considered to have a sufficiently high risk of occlusive vascular events [19].

A meta-analysis from more than 3 million older statin users in 82 studies in over 40 countries showed that adherence was worse among individuals aged more than 75 years than those aged 65-75 years [27]. However, the proportion of secondary prevention subjects who were adherent at follow-up was higher compared to primary prevention subjects. Our analysis suggests that despite overall lessintensive lipid-lowering therapy regimes, elderly patients achieve comparable or even slightly better LDL-C levels than the younger cohort. This suggests that with utilization of available therapy opportunities, reaching treatment goals for LDL-C may be possible in the elderly, also with respect to the 2019 update of the dyslipidemia guidelines by the ESC, suggesting an LDL-C threshold of $<55 \mathrm{mg}$ / $\mathrm{dl}$ for patients with CAD. Due to the limited availability of ezetimibe in the early phases of our analysis and patent protection of rosuvastatin (associated with high costs), the use of these drugs may be underestimated in our analysis. In contrast, with the current availability of generic versions of high-potency statins, including atorvastatin and rosuvastatin, more elderly patients may actually achieve desired LDL-C levels. New lipid-lowering agents, changes in pricing policy, 
and omission of patent protection will lead to a broader use of more potent statin therapy and other agents for intensified lipid-lowering therapy, which will allow for tailored therapy based on the individual's risk profile.

\subsection{Limitations}

The limitations of our study include its retrospective study design with no information regarding previous changes to the lipid-lowering therapy of the patients. Given the retrospective design, assessing cross-sectional patient data, no information on follow-up is available. Moreover, as per the study design, we were not able to properly assess any potential side effects of statin therapy, which may have limited its use in individual patients. Further, due to the loss of patent protection for highly potent statins, including atorvastatin and rosuvastatin, changes in prescription patterns may have impacted therapy after 2016. In addition, the same patients were not evaluated over all three time points. However, long-term follow-up of identical patients would have led to a more pronounced change in patient's age over time, ultimately leading to a decrease in the generalizability of the follow-up cohorts. As admission criteria varied by referring hospital or primary care physician, this may have led to a selection bias from some institutions. However, given the high number of referring physicians and the low number of patients per referring physician in our analysis, this may not have relevantly affected our results. Lastly, our study is based on a predominantly Caucasian cohort; hence, its validity in other cohorts and ethnic groups remains uncertain.

\section{Conclusions}

Evaluating lipid-lowering treatment patterns in a large clinical cohort of patients with known CAD, we observed that patients $\geq 75$ years of age receive lower doses of statin therapy, but reached slightly lower LDL-C levels. However, the majority of elderly patients miss current recommendations regarding LDL-C thresholds. Interestingly, no signs of a higher frequency of statin-induced myopathy in the elderly were observed in our analysis.

Acknowledgements Open Access funding provided by Projekt DEAL.

Author contributions All authors contributed to the study concept and design. Material preparation and data collection and analysis were performed by ID and DW. The first draft of the manuscript was written by ID, and all authors commented on all versions of the manuscript. All authors read and approved the final manuscript. All authors takes responsibility for all aspects of the reliability and freedom from bias of the data presented and their discussed interpretation.

\section{Compliance with ethical standards}

Funding No external funding was used to conduct this work.

Conflict of interest The authors, Iryna Dykun, Daniela Wiefhoff, Matthias Totzeck, Fadi Al-Rashid, R. Alexander Jánosi, Stefanie Hendricks, Tienush Rassaf, and Amir A. Mahabadi, declare that they have no conflict of interest.

Availability of data and material The datasets generated during and/ or analyzed during the current study are not publicly available due to institutional restrictions, but are available from the corresponding author on reasonable request.

Code availability Not applicable.

Open Access This article is licensed under a Creative Commons Attribution-NonCommercial 4.0 International License, which permits any non-commercial use, sharing, adaptation, distribution and reproduction in any medium or format, as long as you give appropriate credit to the original author(s) and the source, provide a link to the Creative Commons licence, and indicate if changes were made. The images or other third party material in this article are included in the article's Creative Commons licence, unless indicated otherwise in a credit line to the material. If material is not included in the article's Creative Commons licence and your intended use is not permitted by statutory regulation or exceeds the permitted use, you will need to obtain permission directly from the copyright holder. To view a copy of this licence, visit http://creativecommons.org/licenses/by-nc/4.0/.

\section{References}

1. Townsend N, Wilson L, Bhatnagar P, Wickramasinghe K, Rayner M, Nichols M. Cardiovascular disease in Europe: epidemiological update 2016. Eur Heart J. 2016;37:3232-45.

2. Fouque D, Roth H, Darne B, Jean-Bouchet L, Daugas E, Drueke $\mathrm{TB}$, et al. Achievement of kidney disease: improving global outcomes mineral and bone targets between 2010 and 2014 in incident dialysis patients in France: the Photo-Graphe 3 study. Clin Kidney J. 2018;11:73-9.

3. Stone NJ, Robinson JG, Lichtenstein AH, Bairey Merz CN, Blum $\mathrm{CB}$, Eckel RH, et al. 2013 ACC/AHA guideline on the treatment of blood cholesterol to reduce atherosclerotic cardiovascular risk in adults: a report of the American College of Cardiology/American Heart Association Task Force on Practice Guidelines. Circulation. 2014;129:S1-45.

4. Afilalo J, Duque G, Steele R, Jukema JW, de Craen AJ, Eisenberg MJ. Statins for secondary prevention in elderly patients: a hierarchical bayesian meta-analysis. J Am Coll Cardiol. 2008;51:37-45.

5. Cannon CP, Blazing MA, Giugliano RP, McCagg A, White JA, Theroux P, et al. Ezetimibe added to statin therapy after acute coronary syndromes. N Engl J Med. 2015;372:2387-97.

6. Sabatine MS, Giugliano RP, Keech AC, Honarpour N, Wiviott SD, Murphy SA, et al. Evolocumab and clinical outcomes in patients with cardiovascular disease. N Engl J Med. 2017;376:1713-22.

7. Giugliano RP, Cannon CP, Blazing MA, Nicolau JC, Corbalan $\mathrm{R}$, Spinar J, et al. Benefit of adding ezetimibe to statin therapy on cardiovascular outcomes and safety in patients with versus without diabetes mellitus: results from IMPROVE-IT (Improved Reduction of Outcomes: Vytorin Efficacy International Trial). Circulation. 2018;137:1571-82. 
8. Naci H, Brugts JJ, Fleurence R, Tsoi B, Toor H, Ades AE. Comparative benefits of statins in the primary and secondary prevention of major coronary events and all-cause mortality: a network meta-analysis of placebo-controlled and active-comparator trials. Eur J Prev Cardiol. 2013;20:641-57.

9. Ofori-Asenso R, Jakhu A, Curtis AJ, Zomer E, Gambhir M, Jaana Korhonen M, et al. A systematic review and meta-analysis of the factors associated with nonadherence and discontinuation of statins among people aged $\% 3 \mathrm{e} /=65$ years. J Gerontol A Biol Sci Med Sci. 2018;73:798-805.

10. Cholesterol Treatment Trialists Consortium. Efficacy and safety of statin therapy in older people: a meta-analysis of individual participant data from 28 randomised controlled trials. Lancet. 2019;393:407-15.

11. Mach F, Baigent C, Catapano AL, Koskinas KC, Casula M, Badimon L, et al. 2019 ESC/EAS Guidelines for the management of dyslipidaemias: lipid modification to reduce cardiovascular risk. Eur Heart J. 2020;41:111-88.

12. European Association for Cardiovascular Prevention, and Rehabilitation, Reiner Z, Catapano AL, De Backer G, Graham I, et al. ESC/EAS Guidelines for the management of dyslipidaemias: the Task Force for the Management of Dyslipidaemias of the European Society of Cardiology (ESC) and the European Atherosclerosis Society (EAS). Eur Heart J. 2011;32:1769-818.

13. Catapano AL, Graham I, De Backer G, Wiklund O, Chapman MJ, Drexel H, et al. 2016 ESC/EAS Guidelines for the management of dyslipidaemias. Eur Heart J. 2016;37:2999-3058.

14. Laufs U, Karmann B, Pittrow D. Atorvastatin treatment and LDL cholesterol target attainment in patients at very high cardiovascular risk. Clin Res Cardiol. 2016;105:783-90.

15. Dykun I, Mahabadi AA, Rassaf TA. A clinical perspective on the 2019 ESC/EAS guidelines for the management of dyslipidaemias: PCSK-9 inhibitors for all? Eur Heart J. 2020. https://doi. org/10.1093/eurheartj/ehaa005.

16. Dykun I, Wiefhoff D, Totzeck M, Al-Rashid F, Janosi RA, Rassaf $\mathrm{T}$, et al. Disconcordance between ESC prevention guidelines and observed lipid profiles in patients with known coronary artery disease. Int J Cardiol Heart Vasc. 2019;22:73-7.

17. Ngo-Metzger Q, Zuvekas S, Shafer P, Tracer H, Borsky AE, Bierman AS. Statin use in the U.S. for secondary prevention of cardiovascular disease remains suboptimal. J Am Board Fam Med. 2019;32:807-17.
18. Kuang Y, Li X, Chen X, Sun H, Tomlinson B, Chan P, et al. Higher prevalence of elevated LDL-C than non-HDL-C and low statin treatment rate in elderly community-dwelling Chinese with high cardiovascular risk. Sci Rep. 2016;6:34268.

19. Stone NJ, Intwala S, Katz D. Statins in very elderly adults (debate). J Am Geriatr Soc. 2014;62:943-5.

20. Schwartz GG, Steg PG, Szarek M, Bhatt DL, Bittner VA, Diaz R, et al. Alirocumab and cardiovascular outcomes after acute coronary syndrome. N Engl J Med. 2018;379:2097-107.

21. Bach RG, Cannon CP, Giugliano RP, White JA, Lokhnygina Y, Bohula EA, et al. Effect of simvastatin-ezetimibe compared with simvastatin monotherapy after acute coronary syndrome among patients 75 years or older: a secondary analysis of a randomized clinical trial. JAMA Cardiol. 2019;4:846-54.

22. Hunt D, Young P, Simes J, Hague W, Mann S, Owensby D, et al. Benefits of pravastatin on cardiovascular events and mortality in older patients with coronary heart disease are equal to or exceed those seen in younger patients: results from the LIPID trial. Ann Intern Med. 2001;134:931-40.

23. Shepherd J, Blauw GJ, Murphy MB, Bollen EL, Buckley BM, Cobbe SM, et al. Pravastatin in elderly individuals at risk of vascular disease (PROSPER): a randomised controlled trial. Lancet. 2002;360:1623-30.

24. Heart Protection Study Collaborative Group. MRC/BHF Heart Protection Study of cholesterol lowering with simvastatin in 20,536 high-risk individuals: a randomised placebo-controlled trial. Lancet. 2002;360:7-22.

25. Savarese G, Gotto AM Jr, Paolillo S, D'Amore C, Losco T, Musella F, et al. Benefits of statins in elderly subjects without established cardiovascular disease: a meta-analysis. J Am Coll Cardiol. 2013;62:2090-9.

26. Dykun I, Mincu R, Hendricks S, Balcer B, Totzeck M, Rassaf T, et al. Efficacy of lipid-lowering therapy beyond statins to prevent cardiovascular events: a meta-analysis. Eur J Prev Cardiol. 2019. https://doi.org/10.1177/2047487319866992.

27. Ofori-Asenso R, Jakhu A, Zomer E, Curtis AJ, Korhonen MJ, Nelson M, et al. Adherence and persistence among statin users aged 65 years and over: a systematic review and meta-analysis. J Gerontol A Biol Sci Med Sci. 2018;73:813-9. 